\title{
A Frequency Reuse-Based Design for Flexible and Scalable Passive Optical Networks (PONs)
}

\author{
Ibrahim M. M. Mohamed \\ Department of Electrical Engineering, Faculty of Engineering, Omar Al-Mukhtar University, Al-beida, Libya
}

\section{Email address:}

engibrahim_2007@yahoo.com

\section{To cite this article:}

Ibrahim M. M. Mohamed. A Frequency Reuse-Based Design for Flexible and Scalable Passive Optical Networks (PONs). Advances in Networks. Vol. 5, No. 1, 2017, pp. 22-30. doi: 10.11648/j.net.20170501.13

Received: June 4, 2017; Accepted: July 14, 2017; Published: October 18, 2017

\begin{abstract}
The accelerated growth in the bandwidth demand emphasizes the necessity to evolve from the currently deployed gigabit-class passive optical networks (PONs) to the next-generation optical access networks (NG-OANs). Different architectures were proposed in the literature in order to create a NG-OAN that is able to fulfilling the aforementioned goal. In this paper, a time-division multiplexing (TDM)/dense wavelength-division multiplexing (DWDM) scheme was proposed. The proposed scheme is sought to satisfy the current and future anticipated bandwidth demands. The architecture we proposed was able to allow different bit rate optical line terminals OLTs to use the same frequency band, and transmit their services over a 24 $\mathrm{km}$ shared feeder to 16 passive remote terminals (PRTs) with $16 \mathrm{ONU}$ group for each. Each group can accommodate up to 16 ONU, total of 256 ONU/PRT, resulting in overall system capacity 4096 ONU. The architecture also allows the independentupgradeability for each optical network terminal ONU.
\end{abstract}

Keywords: Next-Generation Optical Access Networks NG-OAN, TDM-PONs, WDM-PONs, Hybrid TDM/WDM-PONs, Fiber-to-the-Home FTTH, Arrayed Waveguide Grating AWG

\section{Introduction}

Gigabit-class passive optical networks PONs are being deployed in many countries nowadays because of their significant capacity as compared with the traditional copperbased access networks, which allows the delivery of broadband services, such as voice over Internet Protocol (VoIP), and video on demand (VoD). The two main gigabitclass PONs are the Ethernet PON (E-PON, [IEEE802.3ah]) and the gigabit PON (G-PON, [ITU-T G.984]), standardized by the Institute of Electrical and Electronics Engineers (IEEE) and the International Telecommunications Union's Telecommunication Standardization Sector (ITU-T) respectively. Although gigabit-class PONs were envisioned as an ultimate solution to the increased bandwidth demand problem, since they provide an adequate bandwidth segment to each user as compared with the copper-based access networks, their capacity is going to be exhausted as soon as much more bandwidth-hungry services, such as highdefinition television (HDTV), and 3D television (3D-TV) become available in the near future. Thus an evolution from the current PONs to the NG-OANs becomes a matter of urgency. Different architectures were proposed in the literature in order to create a NG-OAN scheme that is able to fulfill the foregoing requirement. For example, IEEE and ITU-T ratified their standards of high speed TDM-PONs, (IEEE 802.3av, [10G-EPON], and ITU-T G.987, [XG-PON]) in 2009 and 2010 respectively [1, 2]. 10G-EPON specifies a symmetric 10-Gbps for both the downstream and upstream traffic, and an asymmetric 10-Gbps for downstream traffic and 1-Gbps for upstream traffic. Similar to the 10G-EPON, XG-PON specifies a symmetric 10 Gbps for both the downstream and upstream traffic. However, it introduces an asymmetric $10 \mathrm{Gbps}$ for downstream traffic and $2.5 \mathrm{Gbps}$ for upstream traffic. Because it is expected for optical links to lose several $\mathrm{dBs}$ of their budget when bit rate increased to 10 Gbps, both the 10G-EPON and XG-PON recommend the use of forward error correction (FEC) to earn extra $\mathrm{dB}$ margin. In all of the aforementioned PONs, service is provided using time division multiplexing (TDM) technology in which a point-to-multipoint (P2MP) connection is established between one optical line terminal (OLT) and several optical network units (ONUs). The main disadvantage of the TDMPONs is their traffic-sharing nature, which poses a real 
challenge to future upgrades. One of the potential candidates for next-generation optical access NGOA is the Hybrid TDM/WDM-PONs. TDM/WDM-PONs were proposed to exploit both, the high speed feature offered by the TDMPONs, and the large number of wavelength counts provided by the WDM-PONs. A hybrid TDM/WDM-PON can be categorized according to the wavelength grid used as either a hybrid TDM/CWDM-PON or a hybrid TDM/DWDM-PON. In the hybrid TDM/CWDM-PON, a 20nm wavelength spacing is used, while in the hybrid TDM/DWDM-PON, a $0.8 \mathrm{~nm}$ or $0.4 \mathrm{~nm}$ wavelength spacing is used. The first commercial colorless gigabit TDM/WDM-PON using a remote protocol terminator was proposed and experimentally demonstrated in [3]. It has been used by Korea Telecom since March 2009. The system was able to provide a high speed fiber-to-the-home service and supporting up to 512 users over a single feeder fiber. The next-generation passive optical network stage 2 (NG-PON2) project was established in 2011 by the full service access network (FSAN) group. The project seeks to exceed 10 Gbps bit rate in the optical access network. The time- and wavelength-division multiplexed passive optical network (TWDM-PON) was chosen in 2012 by the FSAN group as a significant candidate to be the NGPON2 technology. TWDM-PON increases PON data rate by multiplexing multiple PONs. As mentioned above, an XGPON system offers the access rates of $10 \mathrm{Gbps}$ in downstream and $2.5 \mathrm{Gbps}$ in upstream. A scheme of four pairs of wavelengths TWDM-PON is able to multiplexing a four XG-PONs which leads to provide $40 \mathrm{Gbps}$ and $10 \mathrm{Gbps}$ in downstream and upstream, respectively. In this paper, we proposed a new PON scheme. The design comes after studying the characteristics of different optical devices such as, arrayed waveguide gratings (AWGs) and power splitters
(PSs), and the potentiality of incorporating these optical devices together in one architecture for development a flexible and scalable TDM/DWDM-based PON. Incorporating the AWG could realize the frequency re-use approach, which allows for coexistence among different service providers, leads to increases the utilization of the fiber plant and thus increases the efficiency, and facilitates the independent-upgradeability approach. It also leads to enable handling the upstream and downstream traffic through the same input/output ports. The paper is organized as follows: Section 2 provides an overview of AWG architecture and properties. Section 3 presents the proposal PON. Section 4 is devoted for simulation results and discussions. Section 5 concludes the paper.

\section{AWG Architecture and Properties}

An $\mathrm{N} \times \mathrm{N}$ Arrayed Waveguide grating $(\mathrm{N} \times \mathrm{N}$ AWG) is a versatile optical device that can be used as multiplexer, demultiplexer, or router [4-6]. It is a generalization of the $2 \times$ 2 Mach-Zehnder (MZ) interferometer multiplexer. It consists of two identical $\mathrm{N} \times \mathrm{M}$ free propagation regions, also called slabs. The two slabs are connected using an array of $M$ waveguides in such a way that the output ports of the first slab are connected with the input ports of the second slab. The length of any adjacent waveguides in the waveguides array region differs by a constant value $\Delta \mathrm{L}$, which forms a Mach-Zehnder-type grating. For a pure multiplexer, Nin $=\mathrm{n}$ and Nout $=1$, while for a pure demultiplexer, $\mathrm{Nin}=1$ and $\mathrm{N}$ $=\mathrm{n}$, where $\mathrm{n}$ represents the number of wavelengths in an optical signal. In the case of a router, Nin $=$ Nout $=n$. Figure 1 shows a schematic diagram of an $\mathrm{N} \times \mathrm{N}$ AWG.

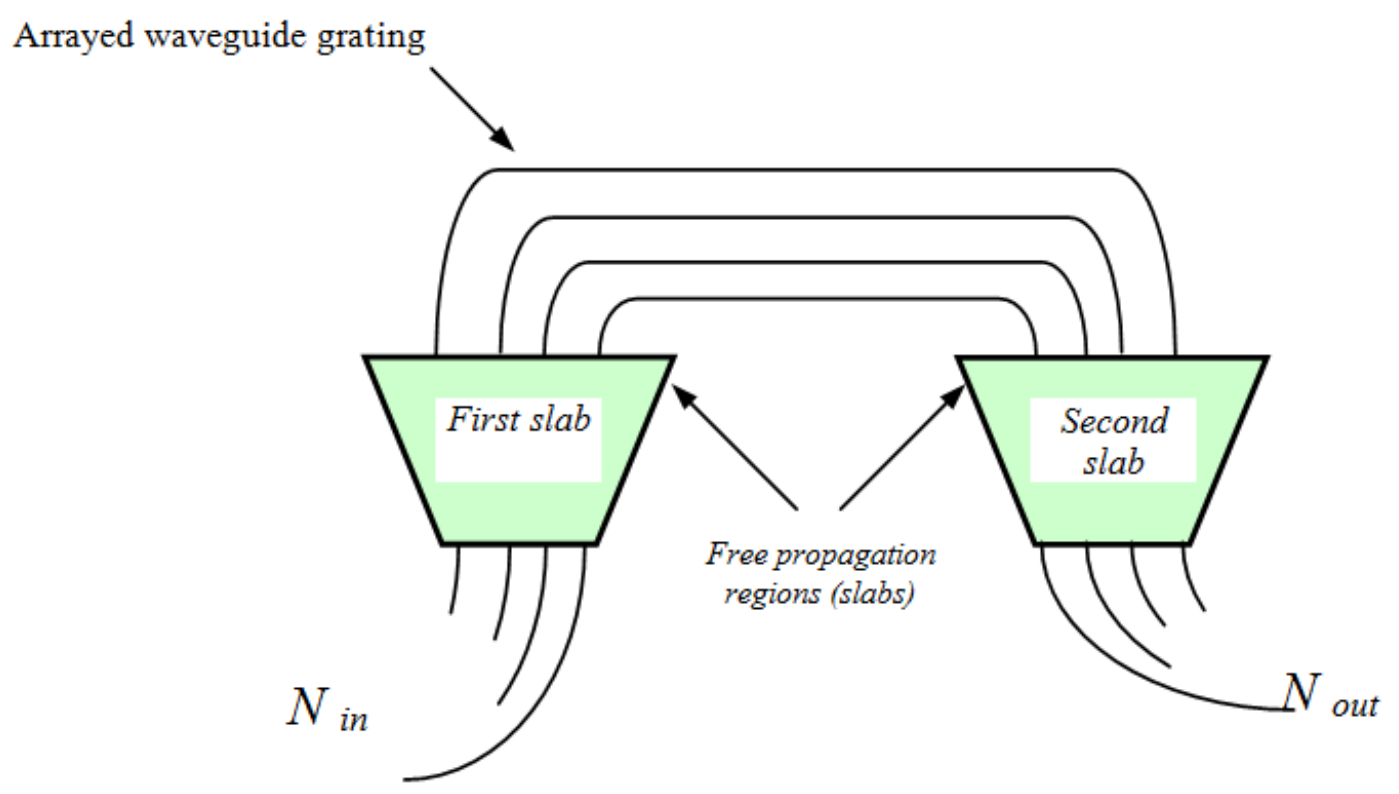

Figure 1. $N \times N A W G$ architecture.

To clarify how AWG works, we consider the situation in which it functions as a demultiplexer. In this case, if an optical signal carrying multiple-wavelengths $(\lambda 1-\lambda n)$, where
$\mathrm{N}=\mathrm{n}$ propagates in the first star coupler (slab), it will arrive at the input of the second star coupler with different phases due to the different waveguide lengths, which results in each 
wavelength being directed to a specific output port. Based on the property of reciprocity, an AWG can also work as a demultiplexer in the opposite direction [7]. Kaneko et al proposed an AWG with stable performance over a wide range of temperatures in one of their previous studies [8]. A silicon-based AWG model with an insertion loss of $1.26 \mathrm{~dB}$ was developed by the company $\mathrm{C} 2 \mathrm{~V}$. However, the main drawback with this model was its large size. Janvit Tippinit and Weerachai Asawamethapant spent considerable efforts and proposed a smaller AWG with a lower insertion loss of
$1.09 \mathrm{~dB}$ [9]. An $\mathrm{N} \times \mathrm{N}$ AWG has two main properties [10]. These properties are the so-called wavelength cyclic property (WCP) and free spectral range property (FSRP), respectively. In WCP, a shift to the input port of the $\mathrm{N} \times \mathrm{N} \quad \mathrm{AWG}$ is followed by an opposite shift to the output port. Whereas in the FSRP, if two different wavelengths incident on a common input port of the $\mathrm{N} \times \mathrm{N}$ AWG, they will exit from a common output port provided that they are separated by the FSR of that $\mathrm{N} \times \mathrm{N}$ AWG. Figure 2 (a) and (b) show schematic diagrams of theses properties.

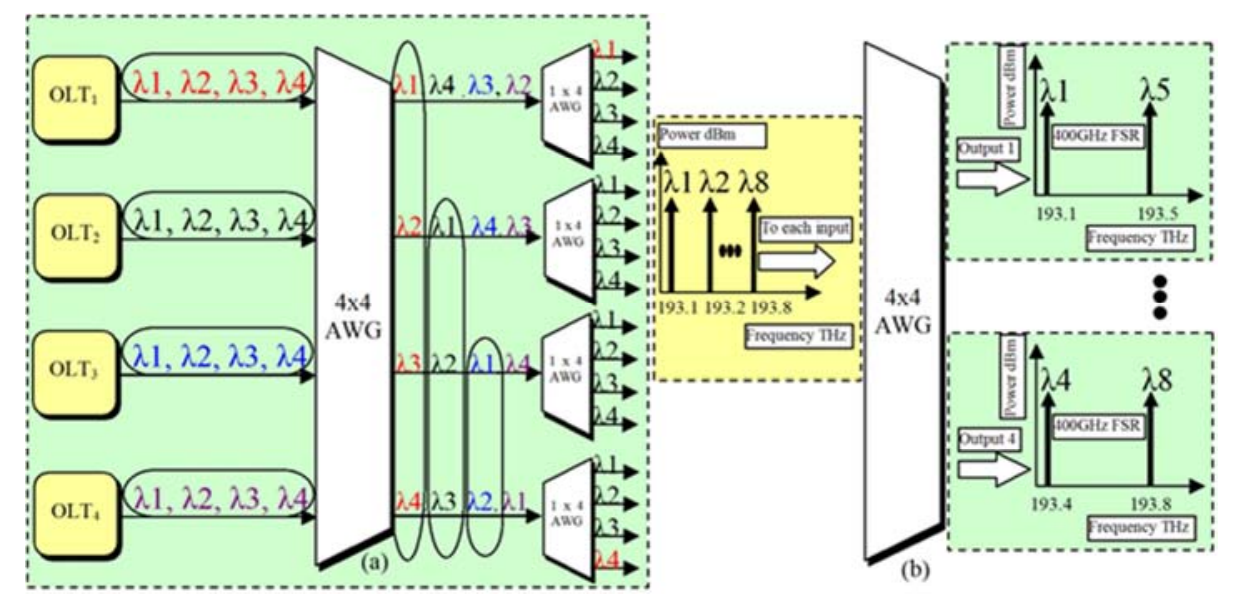

Figure 2. (a) and (b). Schematic diagrams WC and FSR properties.

\section{The Proposed Architecture}

We proposed a hybrid TDM/DWDM scheme. The architecture we proposed which shown in Figure 3 was sought to: create a scalable and flexible PON, satisfy the current and future anticipated bandwidth demands, and increase the efficiency in PON networks. As mentioned above, the design was based on studying the characteristics of different optical devices such as, arrayed waveguide gratings (AWGs), and power splitters (PSs), and the potentiality to incorporate them together in one architecture for development a TDM/DWDM-PON scheme. The AWG was incorporated to exploit its properties of wavelength cyclic (WC), and free spectral range (FSR).

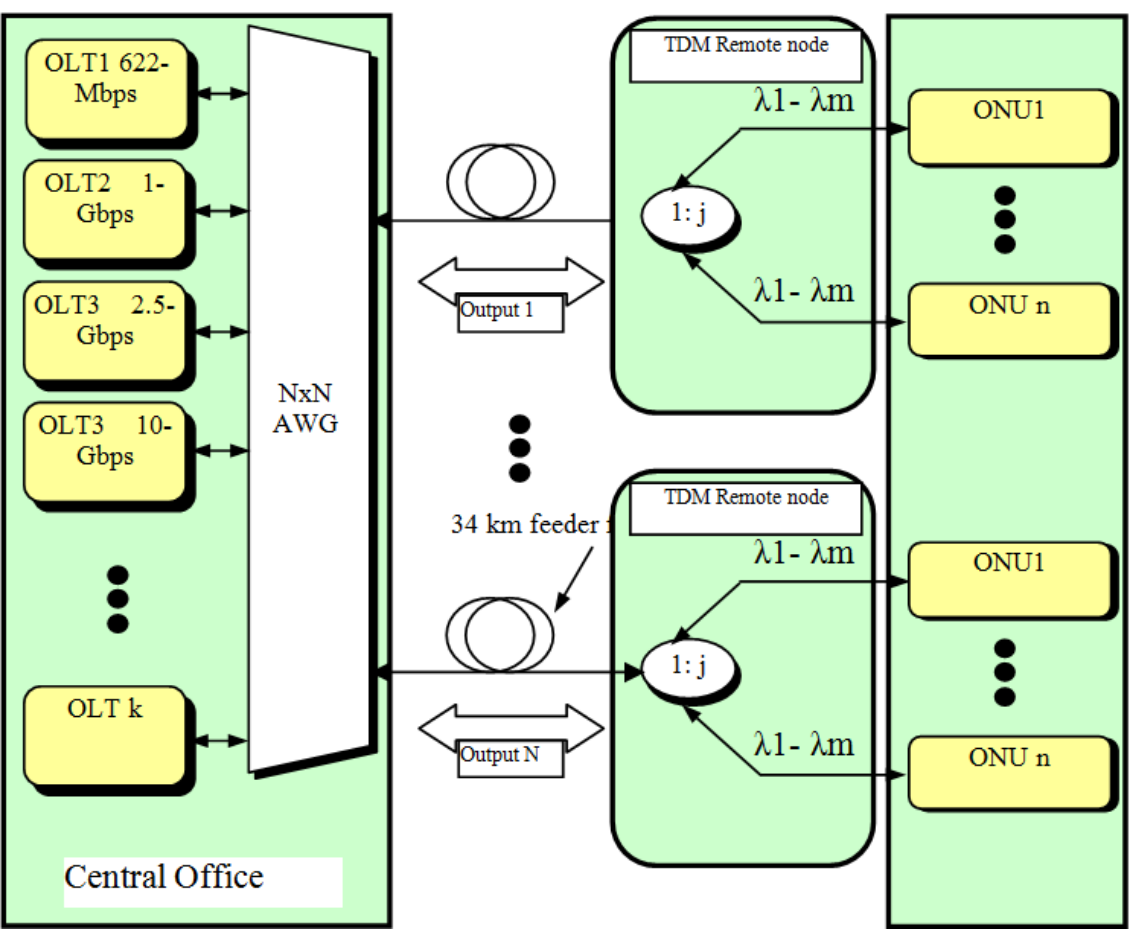

Figure 3. The proposed PON (TDM-based PRT is considered). 
According to the wavelength cyclic property (WCP), if number $\mathrm{k}$ of replicated spectrum $(\lambda 1-\lambda \mathrm{m})$ sent by $\mathrm{k}$ OLTs incident on each input port of an NxN AWG, where $\mathrm{k}=\mathrm{m}=$ $\mathrm{N}$, they will be dimultiplexed through the output ports of the $\mathrm{N} \times \mathrm{N}$ AWG without being overlapped. This would enable several OLTs to handle the same frequency band, which realizes the approach of frequency re-use and thus creates coexistence among different OLTs and increase the utilization of the fiber.

According to the free spectral range property (FSRP), if two different wavelengths incident on a common input port of the $\mathrm{N} \times \mathrm{N}$ AWG, they will exit from a common output port provided that they are separated by the FSR of that $\mathrm{N} \times \mathrm{N}$
AWG. This would enable each ONU to transmit and receive its data via the same input/output ports. Since each ONU in the proposed PON receives a replica of the same aggregation $(\lambda 1-\lambda n)$, and due to each wavelength within the received aggregation refers to different OLT, it becomes possible for each ONU to upgrade its bit rate service independently by using a suitable filter with out affecting the legacy system (achievable flexibility). For further increase in the capacity, we propose to design a hybrid TDM/DWDM-based PRT by incorporating a WDM-DeMux in the PRT unit prior to the power splitter. Figure 4 shows the proposed architecture when hybrid TDM/DWDM-based PRT is considered.

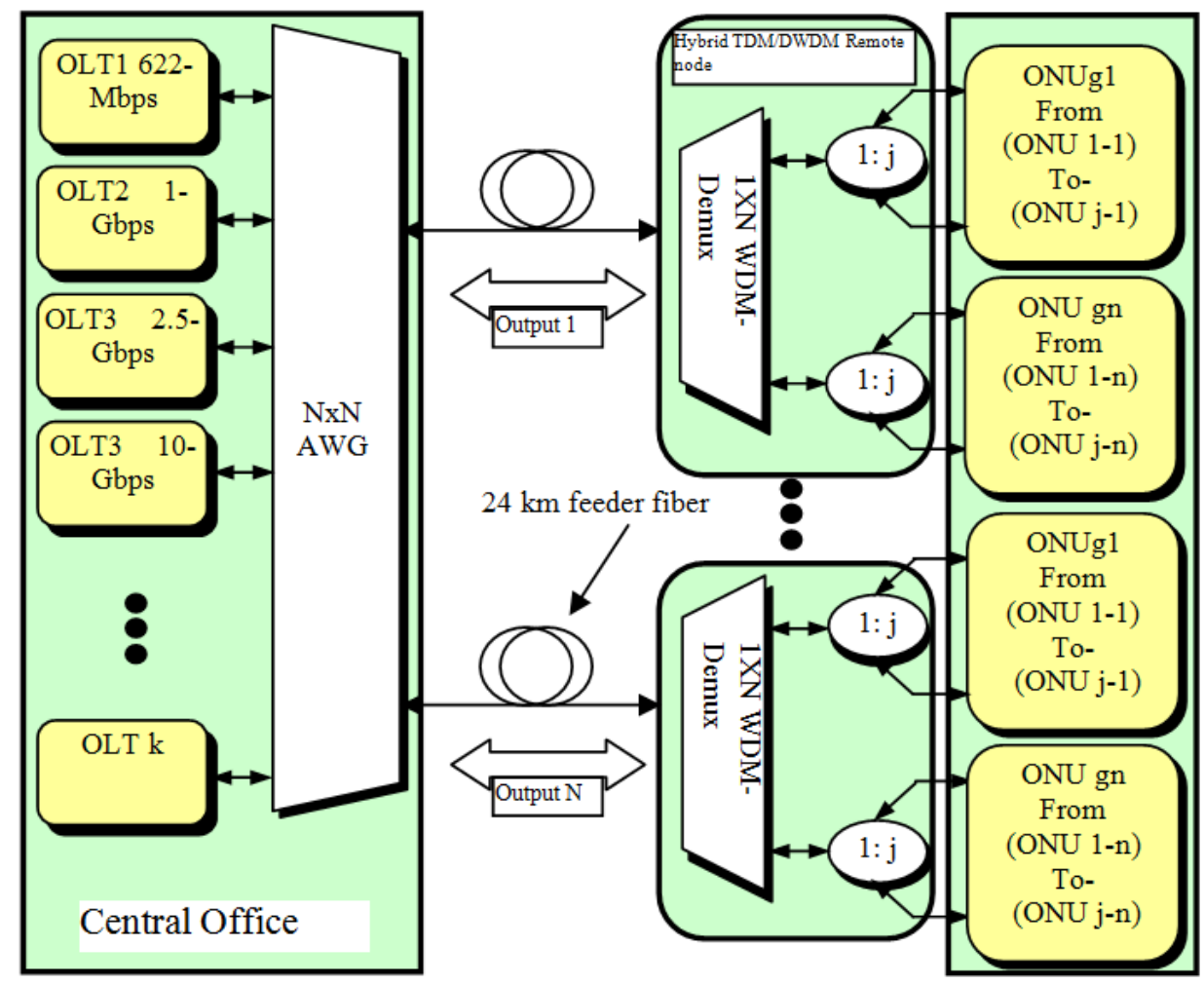

Figure 4. The proposed PON (Hybrid TDM/DWDM-based PRT is considered).

In this case, although it is expected for the capacity of the proposed PON system to increase due to each wavelength will be used by a group of OUN, for example, if 16 wavelength is demultiplexed at the PRT and the power of each is splitted among 16 ONU, the capacity of each ONU group will reach $16 \times 16=256$ ONUs. It is expected for the flexibility of the proposed PON system to reduce, i.e. the architecture will suffers the lack of independentupgradeability, for example, if one subscriber wants to change a PON service, the agreement of the other subscribers under the same wavelength as that of the subscriber is required. However the approach we adopt in designing the PRT leads to avoid such a limitation. In this approach, the PRT was designed in such a way it can support several bit rate PON groups with a specific wavelength for each. Thus, it becomes possible for each ONU to move to another PON group and upgrade its bit rate service by using a suitable filter and performing a simple reconnection at the PRT. Figures 5 (a) and (b) clarify this process where an upgrade from $\lambda 1$ to $\lambda 16$ is assumed. This leads to confirm the necessity of using colorless ONUs. In this context, several proposed colorless ONUs can be found in the literature [11]. As it has been reported that one of the main features that may define a highly scalable new-generation PON is the ability to evolve without affecting legacy systems [12], the concept of scalability of the proposed PON consists in its ability to provide coexistence among different OLTs. It also refers to the increase in the fiber plant utilization. This can be seen obviously at the central office site where several OLTs can share a common fiber exploiting the frequency re-use approach provided by the AWG. Moreover, the increased number of user expected by the proposed PON architecture could also characterize the design as a scalable approach. 

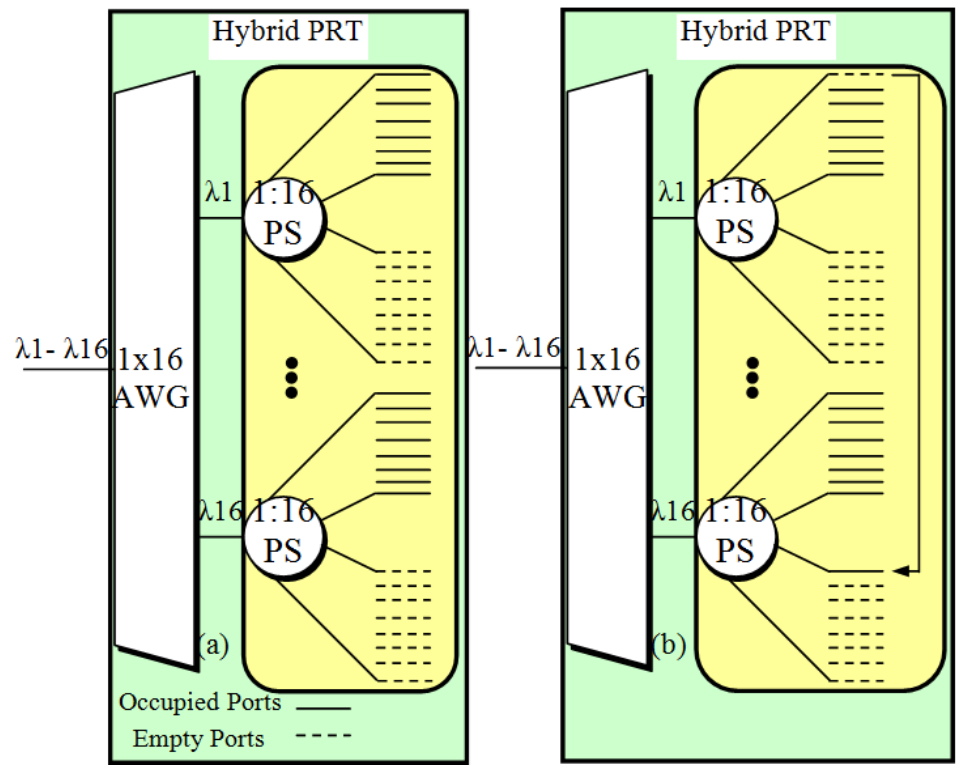

Figure 5. (a) and (b). Upgradeability process corresponding to the proposed architecture.

\subsection{Cost Consideration}

It is expected for the proposed configuration to be a highly cost one due to it is required from each OLT in the architecture to produce an identical multi-wavelength optical signal $(\lambda 1-\lambda m)$. I.e. a number $n$ of optical sources is required in each OLT, which leads to a large number of optical sources being required for the whole system $(\mathrm{m} \times \mathrm{k})$, where $\mathrm{m}$, and $\mathrm{k}$ represent the number of wavelengths in each identical multiwavelength optical signal and the number of OLTs used, respectively. For example if a number of eight OLTs is used, and each of which transmitting an identical multiplewavelength optical signal that contains eight wavelengths, the number of the required optical sources will be 64 , which significantly increase the cost. To reduce the cost of the proposed architecture, a Centralized Wavelength Producing unit CWP was designed and included in the proposed scheme. In the CWP unit, a number of $\mathrm{h}$ centralized optical sources along with a number $\mathrm{i}$ of PSs are used to produce a number of $\mathrm{h} \times \mathrm{i}$ identical aggregations, where $\mathrm{h}=\mathrm{m}$, and $\mathrm{i}=$ $\mathrm{k}$ instead of using $\mathrm{n} \times \mathrm{m}$ optical sources. For example if a number of eight identical multiple-wavelengths signal with eight wavelengths for each are required $(8 \times 8=64$ wavelengths), and it is required for each wavelength to be sent at $0 \mathrm{dBm}$, one can use eight centralized optical wavelength sources with $10 \mathrm{dBm}$ for each along with eight PSs ( $i=8)$ with $1 \mathrm{~dB}$ insertion loss for each according to the following formula

$$
\mathrm{P}_{\text {(source/out) }}=\mathrm{P}_{\text {(centralized/source) }}-10 \log _{10}(\mathrm{j}) \text { - Splitter insertion loss }
$$

Where the term $10 \log _{10}(\mathrm{j})$ represents the splitting loss imposed by a splitter containing $\mathrm{j}$ output ports. Figure 6 shows a CWP unit that has been simulated by using the Opti System software which can centrally produce 8 identical multiple-wavelength optical signals at its output.

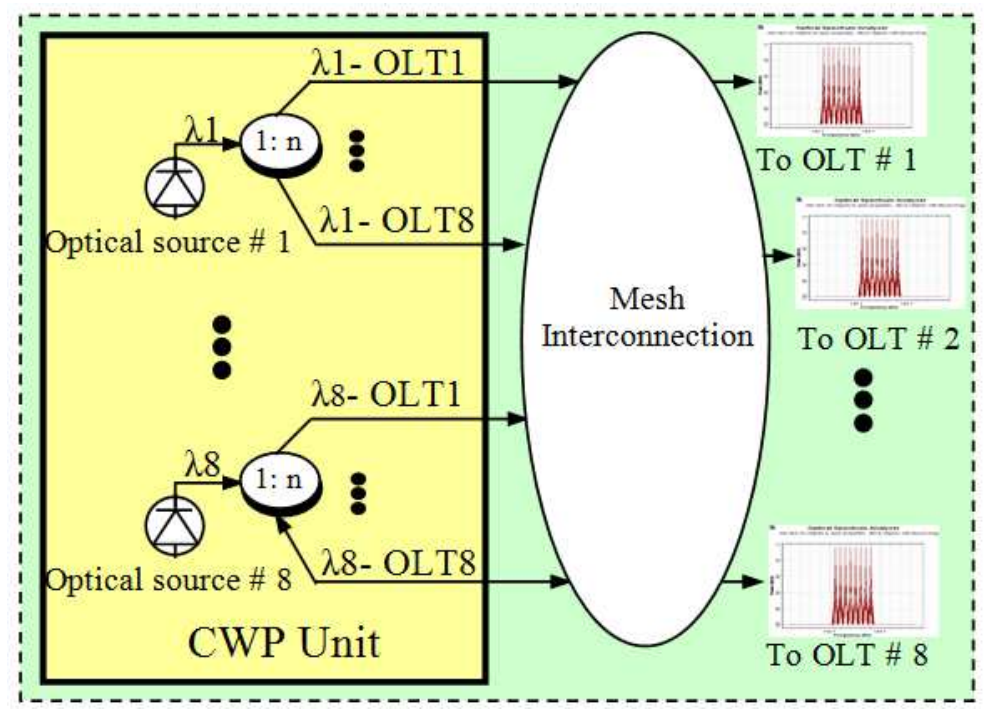

Figure 6. A CWP unit with eight identical multi-wavelength optical signals are considered at its output. 


\subsection{Coexistence with Previous PON Generations}

To discuss the possibility of coexistence with previous generations of PONs in the legacy optical distribution network (ODN), one may look at the wavelength plan options of the TWDM-PON. In fact, there are various options of the TWDM-PON wavelength plan [13]. The first option is based on the re-use of XG-PON wavelength bands. This option defines a finer grid within the already defined bands for XG-PON. As shown in Figure 7 (a), the XG-PON wavelength re-use option is compatible with the G-PON and the radio frequency $(\mathrm{RF})$ video. However, it blocks the standardized XG-PON.

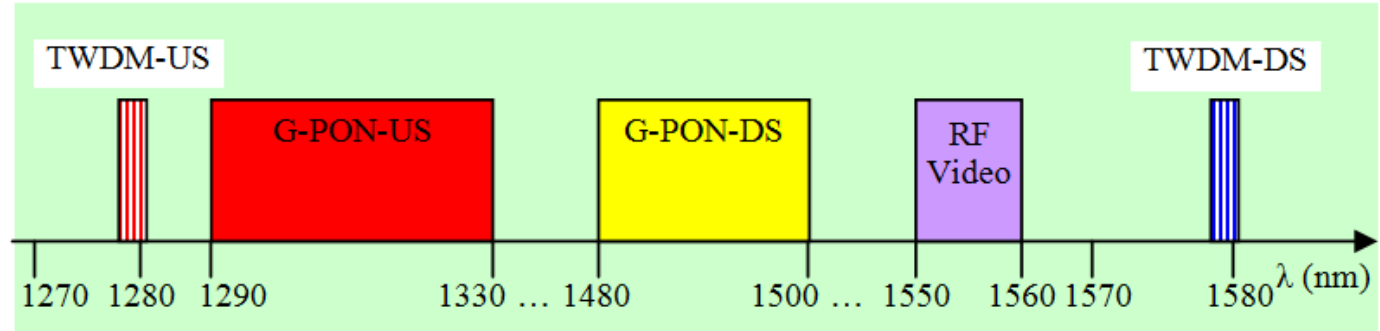

Figure 7. (a). The first option (XG-PON wavelength re-use).

The second option is based on the re-definition of the cband such that it supports both the upstream and downstream wavelengths. As shown in Figure 7 (b), the c-band wavelength plan is compatible with G-PON and XG-PON. However, it blocks the RF video. The third option is based on a mixture of the aforementioned wavelength plans. It defines the L-minus band to support the downstream channels whereas; it defines the C-minus band to support the upstream channels. The upstream channels and the downstream channels are similar to those have been defined in the C-band wavelength plan and the XG-PON wavelength re-use plan, respectively. As shown in Figure 7 (c), the C-minus/L-minus band wavelength plan is compatible to the G-PON and the FR video. However, it blocks the XG-PON downstream channels. Based on the above discussion, and since the proposed architecture was designed in $\mathrm{C}$ band, we deduce that the proposed architecture can allow the coexistence with G-PON and XG-PON in the case in which C-minus/L-minus band wavelength plan is considered.

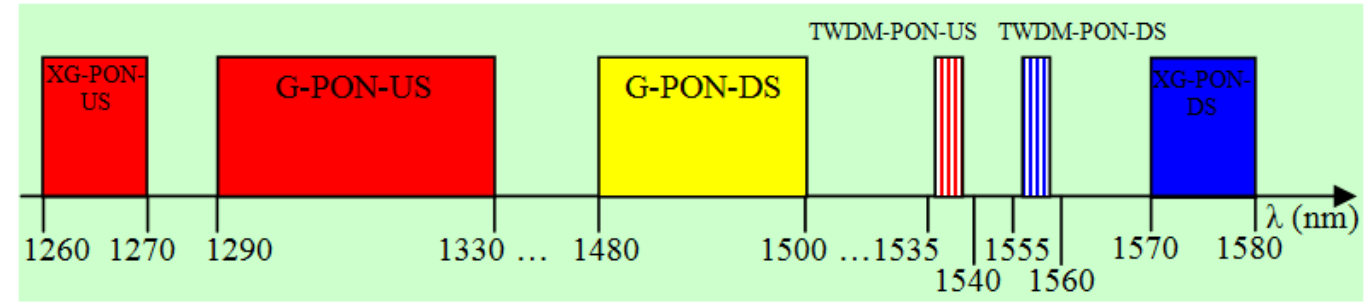

Figure 7. (b). The second option (C-band wavelength plan).

The architecture could allow the coexistence with G-PON, TWDM-PON, and RF video if the XG-PON wavelength reuse plan is consider. It also could allow the coexistence with G-PON, and TWDM-PON if the C-minus/L-minus band wavelength plan is considered. However, a slight change in the wavelength plan of the design is required in the latter case. For example, a shift of $1.5 \mathrm{THz}$ can be suggested making the wavelength plan of the design occupies the range between $191.6 \mathrm{THz}-193.1 \mathrm{THz}$ instead of that have been adopted (193.1 THz - 194.6 THz). Based on the feature of independent-upgradeability that has been discussed in section 3 , the proposed architecture could achieve stacked aggregation of more than 40 Gbps by encouraging each OLT to upgrade its service to $10 \mathrm{Gbps}$. Thus, the confliction with the second wavelength plan option of the TWDM-PON does not seem a real limitation due to the proposed architecture could achieve a bit rate of more than that has been achieved in a TWDM-PON.

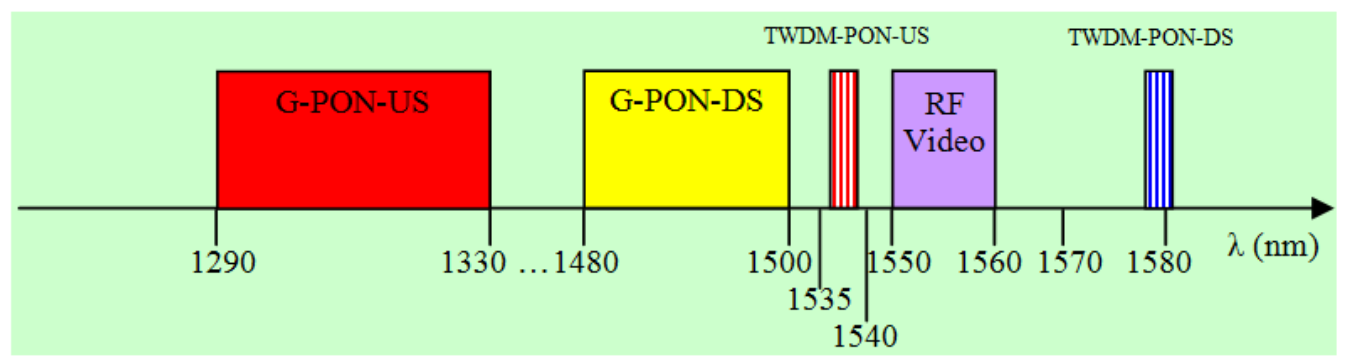

Figure 7. (c). The third option (C-minus/L-minus wavelength plan). 


\section{Simulation Results and Discussions}

Tow simulations were conducted in this study. In the first simulation, results were obtained where a TDM-based PRT is considered, whereas in the second simulation results were obtained where a hybrid TDM/DWDM-based PRT is considered. In both simulations, four different bit rate OLTs are considered.

\subsection{First Simulation (TDM-Based PRT Is Considered)}

This part of simulation is divided into two subsections. In the first subsection, three different bit rate OLTs are considered (622 Mbps, $1 \mathrm{Gbps}$, and $2.5 \mathrm{Gbps}$ ). While in the second subsection, 10 Gbps OLT is included.

\subsubsection{TDM-based PRT (622 Mbps, 1 Gbps, and 2.5 Gbps Bit Rates Are Considered)}

In this part of simulation, three different bit rate OLTs were employed, each of which has a unique NRZ Pseudo data, (622 Mbps, 1 Gbps and 2.5 Gbps). Theses NRZ Pseudo Random data were transmitted simultaneously at $0 \mathrm{dBm}$. A $16 \times 16$ AWG with $1.26 \mathrm{~dB}$ insertion loss and $100 \mathrm{GHz}$ frequency spacing was assigned. A $1 \times 16$ Power Splitter with $14.04 \mathrm{~dB}$ Splitting/insertion loss was assigned. A frequency range of 1.5 $\mathrm{THz}$ with $100 \mathrm{GHz}$ frequency spacing $(193.1 \mathrm{THz}-194.6$ $\mathrm{THz}$ ), which represents a multiple-wavelength optical signal that carries sixteen wavelengths was allocated for each OLT. Variable length single-mode fiber (feeder fiber) with 0.2 $\mathrm{dB} / \mathrm{km}$ attenuation and $16.75 \mathrm{ps} /(\mathrm{nm} . \mathrm{km})$ dispersion was considered. A P-type intrinsic N-type (PIN) photodiode was used for signal reception due to its low biasing voltage and low cost. A BER of $10^{-9}$ was chosen as a reference for operational requirement. Figure 8 shows BER versus feeder fiber at $0 \mathrm{dBm}$ and three different bit rates, $622 \mathrm{Mbps}, 1 \mathrm{Gbps}$ and 2.5 Gbps. As can be seen obviously in the graph, the BER increases as feeder fiber increases until it reaches $10^{-9}$ at $34 \mathrm{~km}$ and 2.5 Gbps, $37 \mathrm{~km}$ and $1 \mathrm{Gbps}$, and $39 \mathrm{~km}$ and $622 \mathrm{Mbps}$, and then continuous to increase as the feeder fiber increases. Based on this result, we conclude that transmission is possible over the proposed PON until $34 \mathrm{~km}$ because it is the lowest distance obtained for the simultaneous transmission.

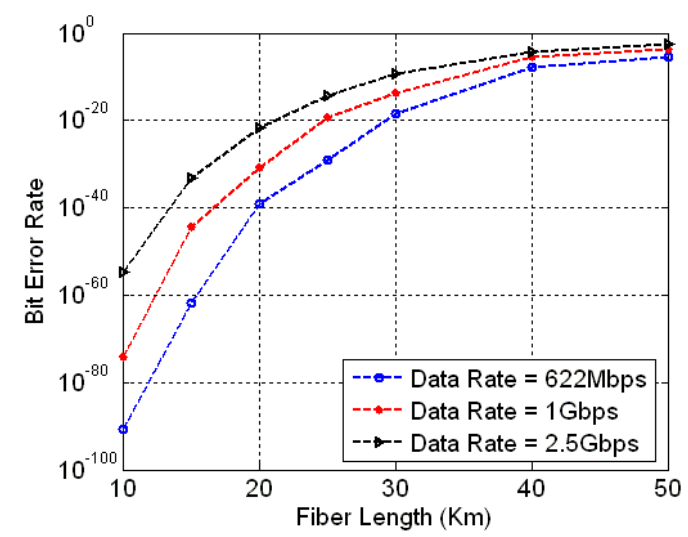

Figure 8. BER versus fiber length at $0 \mathrm{dBm}$ and different Bit rates (TDMbased PRT is considered).

\subsubsection{TDM-Based PRT (10 Gbps Is Included)}

In this part of simulation, a $10 \mathrm{Gbps}$ OLT was included. To compensate for losses expected due to the increased bit rate, we decided to: increase the input power to $2 \mathrm{dBm}$, use an optimized gain Avalanche Photodiode (APD) instead of PIN photodiode for signal reception, and recommend the use of forward error correction code (FEC). A BER of $2.9 \times 10^{-4}$ was chosen as a reference for operational requirement because it represents the pre-FEC BER required to achieve a post-FEC BER lower than $10^{-9}$. Figure 9 shows BER versus APD gain (M) at $10 \mathrm{Gbps}, 34 \mathrm{~km}$ feeder, and $2 \mathrm{dBm}$, where a BER value of $2.78 \times 10^{-5}$ which is lower than that is required for the pre-FEC was achieved at $\mathrm{M}=8$.

\subsection{Second Simulation (Hybrid TDM/DWDM-Based PRT Is Considered)}

Similar to section 5.1, this part of simulation is divided into two subsections. In the first subsection, three different bit rate OLTs are considered (622 Mbps, $1 \mathrm{Gbps}$, and $2.5 \mathrm{Gbps}$ ). While in the second subsection, 10 Gbps OLT is included.

\subsubsection{Hybrid TDM/DWDM-Based PRT (622 Mbps, 1 Gbps, and 2.5 Gbps Bit Rates Are Considered)}

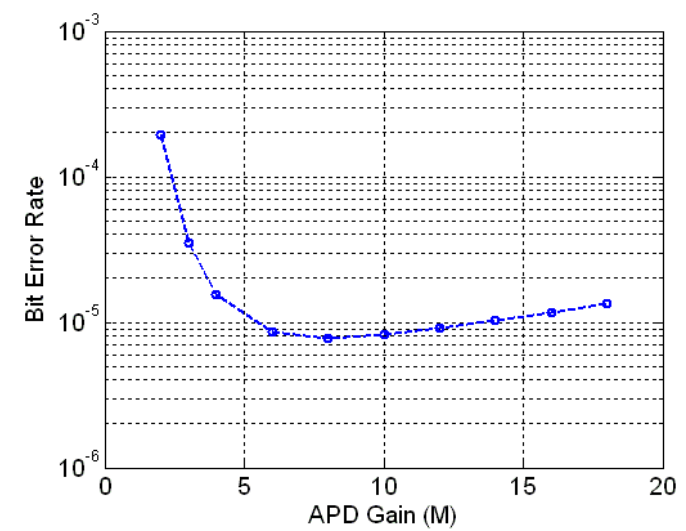

Figure 9. BER versus APD gain (M) at $10 \mathrm{Gbps}, 34 \mathrm{~km}$, and $2 \mathrm{dBm}$ (TDMbased PRT is considered).

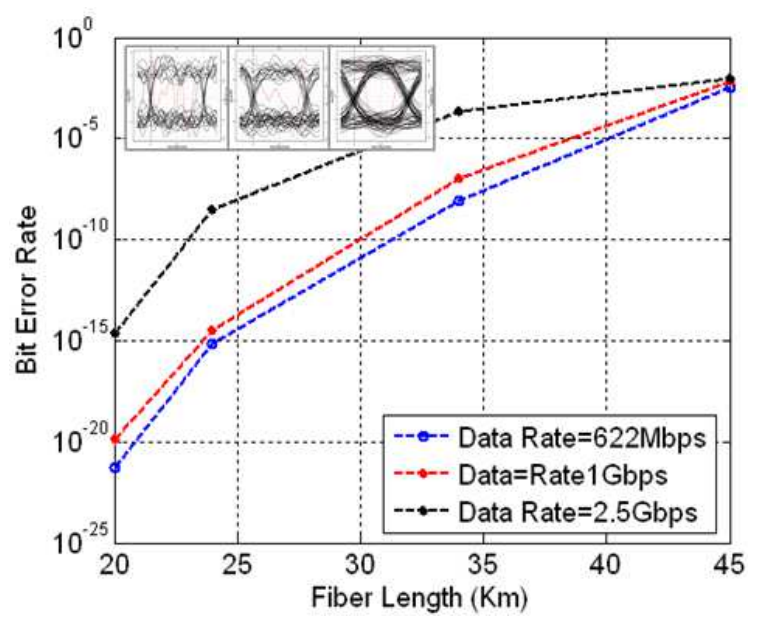

Figure 10. BER versus fiber length at $0 \mathrm{dBm}$ and different Bit rates (Hybrid TDM/DWDM-based PRT is considered). 
In this part of simulation, all components and parameters used are identical with that have been used in section 5.1.1. Similarly, three different bit rate OLTs were employed, (622 Mbps, 1 Gbps and 2.5 Gbps). A WDM-Demux with $1.26 \mathrm{~dB}$ insertion was incorporated in the PRT prior to the PS. Figure 10 shows BER versus feeder fiber at $0 \mathrm{dBm}$ and three different bit rates, $622 \mathrm{Mbps}, 1 \mathrm{Gbps}$ and $2.5 \mathrm{Gbps}$. As can be seen obviously in the graph, the BER increases as feeder fiber increases until it reaches $10^{-9}$ at $24 \mathrm{~km}$ and $2.5 \mathrm{Gbps}, 30$ $\mathrm{km}$ and $1 \mathrm{Gbps}$, and $33 \mathrm{~km}$ and $622 \mathrm{Mbps}$, and then continuous to increase as the feeder fiber increases. Based on this result, we conclude that transmission is possible over the proposed PON until $24 \mathrm{~km}$ because it is the lowest distance obtained for the simultaneous transmission.

\subsubsection{Hybrid TDM/DWDM-Based PRT (10 Gbps Is Included)}

In this part of simulation, all components and parameters used are identical with that have been used in section 5.1.2. Similarly, a 10 Gbps OLT was included. A WDM-Demux with $1.26 \mathrm{~dB}$ insertion was incorporated in the PRT prior to the PS. Figure 11 shows BER versus APD gain (M) at 10 Gbps, $24 \mathrm{~km}$ feeder, and $2 \mathrm{dBm}$, where a BER value of $2.78 \times 10^{-5}$ which is lower than that is required for the preFEC was achieved at $M=6$.

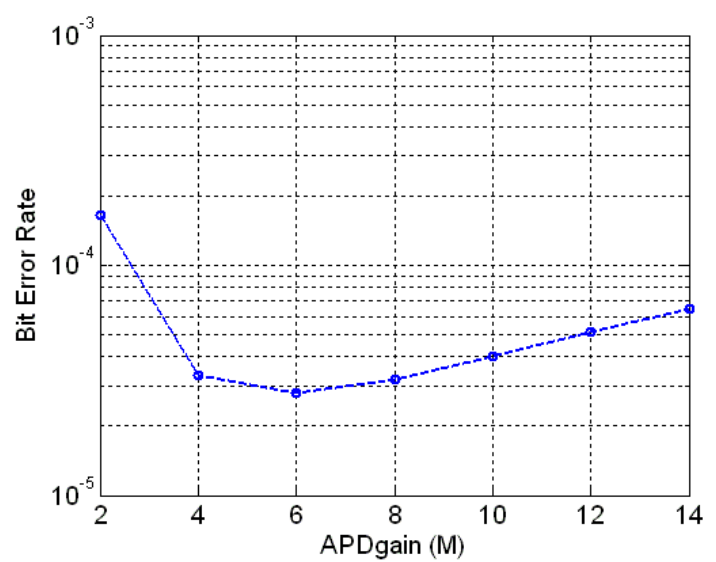

Figure 11. BER versus APD gain (M) at $10 \mathrm{Gbps}, 24 \mathrm{kmd}$, and $2 \mathrm{dBm}$ (Hybrid TDM/DWDM-based PRT is considered).

\section{Conclusions and Future Work}

In this paper, we proposed a hybrid TDM/DWDM-PON scheme. The architecture we proposed was able to allow different bit rate OLTs (622 Mbps, $1 \mathrm{G}$ bps, $2.5 \mathrm{Gbps}$, and 10 Gbps) to use a common frequency band, and transmit their services over a $24 \mathrm{~km}$ shared feeder to 16 passive remote terminals (PRTs) with 16 ONU group for each. Each group can accommodate up to $16 \mathrm{ONU}$, total of $256 \mathrm{ONU} / \mathrm{PRT}$, resulting in overall system capacity 4096 ONU. Incorporating AWG offers the following opportunities: (A). The architecture enables each ONU to handle its downstream and upstream traffic through the same input/output ports. (B). The architecture is able to allow different bit rate OLTs to use the same frequency band and handle their traffic over a common fiber (Achievable Co-Existence, and Increasable Fiber Utilization). (C). Since each received wavelength refers to an individual OLT, each ONU can move to higher bit rate service simply by using a suitable filter and simple reconnection with out affecting the legacy ones (Achievable Independent-Upgradeability). This feature of Independentupgradeability encourages each OLT in the proposed TDM/DWDM-PON to upgrade its bit rate service to 10 Gbps, which leads to achieve a stacked bit rate of more than 40 Gbps. The architecture was designed to work in $\mathrm{C}$ band (1530-1560 nm), which allows for co-existence with the commercially deployed G-PONs, and XG-PONs as they were characterized as working in different band segments. i.e. $([1290-1330] \mathrm{nm} /[1480-1500] \mathrm{nm}$, and [1260-1270] nm / [1570-1580] nm for G-PONs, and XG-PONs US/DS transmission, respectively. Moreover, working in the $\mathrm{C}$ band allows the use of the erbium-doped fiber amplifiers EDFAs if future necessity arises. As mentioned above, the Independent-upgradeability feature leads to achieve a stacked aggregation of $40 \mathrm{Gbps}$ or more, which is similar or higher than that has been achieved in the TWDM-PON. Thus, the confliction with the wavelength plan of the TWDM-PON does not seem a real limitation. As mentioned above, working in the $\mathrm{C}$ band allows the use of the EDFAs. Since EDFAs are protected against the cross-talk effect in the case in which a simultaneous amplification is required, provided that a frequency of $10 \mathrm{KHz}$ or higher is used for frequency spacing, which suits our design where $100 \mathrm{GHz}$ was chosen for DWDM spacing, a future plan on this topic is to incorporate the EDFA in the design for further increase in the transmission distance and number of accommodated ONUs. However incorporating such amplifiers is not straight forward, i.e. they should be optimized before being incorporated in the design. Thus our future plan will include the optimization of the EDFA in terms of its power pump, EDF length, overlap factor, erbium ions concentration, and configuration \& pumping wavelength used.

\section{References}

[1] Keiji Tanaka et al, "IEEE 802.3av 10G-EPON Standardization and Its Research and Development Status," Journal of Lightwave Technology, vol. 28, pp. 651-661, 2010.

[2] ITU-T Recommendation G.987 series, "10-Gigabit-capable passive optical network (XG-PON) systems,” 2010.

[3] Jie Hyun Lee, at al. "First Commercial Deployment of a Colorless Gigabit WDM/TDM Hybrid PON System Using Remote Protocol Terminator," Lightwave Technology, Journal of, vol. 28, pp. 344-351, 2010.

[4] M. K. Smit, "New focusing and dispersive planar component based on an optical phased array," Electron. Lett, vol. 24, pp. 385- 86, 1988.

[5] Takahashi et al, "Polarization-Insensitive Arrayed-Waveguide Wavelength Multiplexer with Birefringence Compensating Film". IEEE PHOTONICS TECHNOLOGY LE'ITERS, VOL. 5, NO. 6, JUNE 1993. 
[6] Inoue et al, "Polarization mode converter with polyimide half waveplate in silica-based planar lightwave circuits," IEEE Photon. Technol. Lett, vol. 6, pp. 626628, 1994.

[7] C. R. Giles, M. Newhouse, J. Wright, and K. Hagimoto, "Special Issue on System and Network Applications of Optical Amplifiers," J. Lightwave Tech, vol. 13, May 1995.

[8] A. Kaneko, S. Kamei, Y. Inoue, H. Takahashi, and A. Sugita, "Athermal silica-based arrayed-waveguide grating (AWG) multi/demultiplexers with new low loss groove design," Elect. Lett, vol. 36, no. 4, pp 318-319, Feb. 2000.

[9] Tippinit J and Asawamethapant W, "Optical properties improvement on A WG Achieved by Adding Transmission Star Couplers into FPRl" Electrical Engineering/Electronics, Computer, Telecommunications and Information Technology (ECTI-CON), 2012 9th International Conference, 16-18 May 2012, 978-1-4673-2025-2112/\$31.00 C2012 IEEE.
[10] A. Kaneko, T. Goh, H. Yamada, T. Tanaka, and L. Ogawa, "Design and applications of silica-based planar lightwave circuits," IEEE Journal of Selected Topics in Quantum Electronics, vol. 5, no. 5, pp 1227-1236, Sept/Oct. 1999.

[11] Josep Prat et al, "Next-Generation FTTH Passive Optical Networks", Springer Science, Business Media B. V, 2008.

[12] J. i. Kani, "Enabling Technologies for Future Scalable and Flexible WDM-PON and WDM/TDM-PON Systems," IEEE Journal of Selected Topics in Quantum Electronics, vol. 16, pp. 1290-1297, 2010.

[13] Yuanqiu Luo et al, "Time - and Wavelength-Division Multiplexed Passive Optical Network (TWDM-PON) for Next-Generation PON Stage 2 (NG-PON2)", JOURNAL OF LIGHTWAVE TECHNOLOGY, VOL. 31, NO. 4, FEBRUARY 15, 2013. 